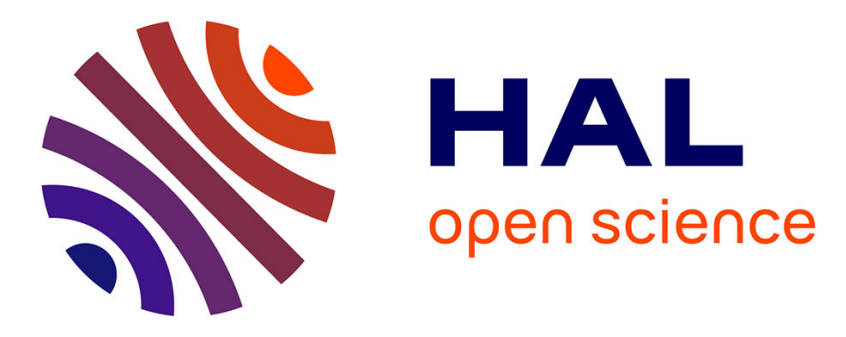

\title{
Modeling protein-mediated morphology in biomembranes
}

\author{
Ashutosh Agrawal, David Steigmann
}

\section{To cite this version:}

Ashutosh Agrawal, David Steigmann. Modeling protein-mediated morphology in biomembranes. Biomechanics and Modeling in Mechanobiology, 2009, 8 (5), pp.371-379. hal-00771715

\section{HAL Id: hal-00771715 \\ https://hal.science/hal-00771715}

Submitted on 9 Jan 2013

HAL is a multi-disciplinary open access archive for the deposit and dissemination of scientific research documents, whether they are published or not. The documents may come from teaching and research institutions in France or abroad, or from public or private research centers.
L'archive ouverte pluridisciplinaire HAL, est destinée au dépôt et à la diffusion de documents scientifiques de niveau recherche, publiés ou non, émanant des établissements d'enseignement et de recherche français ou étrangers, des laboratoires publics ou privés. 


\title{
Modeling protein-mediated morphology in biomembranes
}

\author{
Ashutosh Agrawal · David J. Steigman
}

\begin{abstract}
The equilibrium theory for lipid membranes is used to describe the structure of nuclear pores and the membrane shapes accompanying endocytosis. The commonly used variant of the theory contains a fixed parameter called the spontaneous curvature which accounts for asymmetry in the bending response of the membrane. This is replaced here by a variable distribution of spontaneous curvature representing the influence of attached proteins. The required adjustments to the standard theory are described and the resulting model is applied to the study of membrane morphology at the cites of protein-assisted nuclear pore formation and endocytosis.
\end{abstract}

Keywords Biomembranes - Shape equation - Variable spontaneous curvature

\section{Introduction}

Lipid bilayers are fundamental components of cell structures that strongly influence cell function. Bilayers are composed of transversely oriented lipid molecules containing hydrophilic head groups and hydrophobic tails (Ou-Yang et al. 1999). These groups are arranged in opposing orientations that effectively shield the tail groups from the surrounding aqueous solution. Relative misalignment of the lipids entails an energetic cost which manifests itself as flexural

\author{
A. Agrawal \\ Department of Civil and Environmental Engineering, \\ University of California, Berkeley, CA 94720, USA \\ D. J. Steigmann $(\varangle)$ \\ Department of Mechanical Engineering, University of California, \\ Berkeley, CA 94720, USA \\ e-mail: steigman@me.berkeley.edu
}

stiffness of the film, while the bilayer is otherwise fluid-like in its response. Further, bilayers are naturally symmetric with respect to a midsurface and so lack a natural orientation. Kim et al. (1998) have demonstrated that proteins play a principal role in the morphology of lipid bilayers in which they are embedded. In particular, these authors have shown that transmembrane proteins which are roughly conical in shape, with the vertex lying to one side of the membrane, induce membrane bending as a consequence of local protein-membrane interactions. These interactions effectively break the natural bilayer symmetry, producing a natural curvature of the membrane over patches of surface containing many similar proteins. We propose a model for bilayers containing such a high areal density of proteins that they may be effectively regarded as being continuously distributed over the membrane surface. Here, the natural curvature induced on the local scale is modeled in terms of the so-called spontaneous curvature, a parameter that is well known in the mathematical theory of membrane equilibria (Helfrich 1973; Ou-Yang et al. 1999). In the conventional theory the spontaneous curvature is a fixed parameter. However, we assume the spontaneous curvature to vary spatially over the surface in a manner that reflects the influence of the proteins. Thus, we suppose the spontaneous curvature to be a function of protein type and areal concentration; the greater the concentration of a particular type, the greater the magnitude of spontaneous curvature. The sign of the spontaneous curvature reflects the orientation of proteins relative to the surface normal. Such a dependence has yet to be quantified precisely. A plausible assumption is that spontaneous curvature is proportional to protein concentration, with the sign of the constant of proportionality being that of the natural curvature induced by local protein geometry.

The model is illustrated by computing membrane shapes in two examples of protein-mediated morphology. In the first, membrane shape in the vicinity of an isolated nuclear 
pore is studied. These shapes resemble the interior sectors of axisymmetric tori connected to parallel planes at their apices. Nuclear pores play a central role in the structure and function of the cell nucleus (Boal 2002). In the second example, the shape of the membrane in the process of protein-assisted endocytosis is predicted. Typically, endocytosis entails the creation of a bud on the membrane which facilitates encapsulation and transport of various substances into and out of the cell. Often, this process is assisted by attached proteins such as clathrin which self-assemble to form a coating on one side of the membrane. This structure is not attached directly to the membrane, but rather to discrete transmembrane receptors which maintain congruence of the coat and membrane while leaving the underlying membrane structure undisturbed (Boal 2002). The protein coat induces a natural curvature in the membrane but does not affect it flexural stiffness or its fluidity in the two-dimensional sense.

In principle, a model of the kind envisaged should account for the dynamic two-way coupling between protein distribution/concentration and membrane shape. We foresee a framework that accounts for the diffusion of protein species coupled to membrane shape via, for example, an evolving distribution of spontaneous curvature. Such a model might have the potential to offer insights into the morphologies of protein-rich structures such as the endoplasmic reticulum. As a preliminary step in the development of such a framework, the simpler one-way coupling problem of finding the equilibrium shapes of membranes in the presence of an assigned spontaneous curvature distribution is solved in the present paper. For this purpose we adapt the general theory of lipid membranes, regarded as fluid surfaces with bending elasticity (Steigmann 1999a). This theory accommodates a completely general dependence of membrane free energy on the mean and Gaussian curvatures of the surface and accommodates non-uniform properties.

Section 2 contains a summary of the model of biomembranes from the viewpoint of elastic shell theory. The essential difference between biomembranes and conventional shells is the material symmetry underlying the dependence of the relevant free-energy function on the strain and curvature. A framework for extending the conventional concept of fluidity from three-dimensional continua to surfaces with bending and stretching resistance has been developed in Steigmann (1999a). This yields the theory of biomembranes, traditionally based on variational arguments (Ou-Yang et al. 1999), directly from the balance and constitutive equations of nonlinear shell theory (Steigmann 1999a,b). To connect this approach to that adopted in the literature, we outline the variational derivation of the model in Sect. 3. This generalizes the conventional development to non-uniform biomembranes and accommodates non-uniform spontaneous curvature as a special case. In Sect. 4 we specialize the model to describe surfaces of revolution. This specialization yields ordinary differential equations amenable to simple analysis. These are used in Sects. 5 and 6, respectively, to predict the equilibrium shape of an isolated nuclear pore and of an isolated bud formed during endocytosis.

\section{Equilibrium equations for biomembranes}

We denote the membrane surface by $\omega$. Position on this surface, relative to a specified origin, is described in parametric form by the function $\mathbf{r}\left(\theta^{\mu}\right)$, where $\theta^{\mu} ; \mu=1,2$, are surface coordinates. Here and henceforth Greek indices range over $\{1,2\}$ and, if repeated, are summed over that range. We assume familiarity with tensor notation and with the concepts of contra- and co-variance (Kreyszig 1959). Subscripts preceded by commas indicate partial derivatives with respect to the coordinates, while those preceded by semicolons indicate covariant derivatives. The surface coordinates induce the basis $\mathbf{a}_{\alpha}=\mathbf{r}_{, \alpha}$ for the tangent plane to $\omega$ at the point with coordinates $\theta^{\mu}$. The induced metric is $a_{\alpha \beta}=\mathbf{a}_{\alpha} \cdot \mathbf{a}_{\beta}$, and is assumed to be positive definite. A dual basis on the tangent plane is then given by $\mathbf{a}^{\alpha}=a^{\alpha \beta} \mathbf{a}_{\beta}$, where $\left(a^{\alpha \beta}\right)=$ $\left(a_{\alpha \beta}\right)^{-1}$. The orientation of the surface is defined locally by the unit-normal field $\mathbf{n}=\mathbf{a}_{1} \times \mathbf{a}_{2} /\left|\mathbf{a}_{1} \times \mathbf{a}_{2}\right|$, and its local curvature by the covariant surface-tensor field

$b_{\alpha \beta}=\mathbf{n} \cdot \mathbf{r}_{, \alpha \beta}=-\mathbf{a}_{\alpha} \cdot \mathbf{n}_{, \beta}$.

If $\boldsymbol{v}$ and $\mathbf{t}$ are orthonormal vectors on the tangent plane, then the normal curvatures and the twist on these axes are given respectively by

$\kappa_{\nu}=b_{\alpha \beta} \nu^{\alpha} \nu^{\beta}, \quad \kappa_{t}=b_{\alpha \beta} t^{\alpha} t^{\beta}$ and $\tau=b_{\alpha \beta} v^{\alpha} t^{\beta}$.

Let $a=\operatorname{det}\left(a_{\alpha \beta}\right)$, and let $A$ be the value of $a$ on a fixed reference surface $\Omega$. If the coordinates $\theta^{\mu}$ are conceived of as being convected with the deformation from $\Omega$ to $\omega$, then the local ratio, $J$, of material area after deformation to that before is $J=\sqrt{a / A}$. If $\rho$ and $\rho_{0}$ are the areal mass densities (mass per unit surface area) on $\omega$ and $\Omega$, respectively, then

$J=\rho_{0} / \rho$.

Mass is conserved, as we assume here, if and only if $\rho_{0}$, when expressed as a function of $\theta^{\mu}$ and time, is independent of the latter; i.e., $\rho_{0}=\rho_{0}\left(\theta^{\mu}\right)$.

The fundamental differential equations of equilibrium are the shape equation (Ou-Yang et al. 1999; Steigmann 1999a) and an equation restricting the variation of the surface tension with surface coordinates. These are (Steigmann 1999a)

$$
\begin{aligned}
p=\Delta & \left(\frac{1}{2} \rho F_{H}\right)+\left(\rho F_{K}\right)_{; \alpha \beta} \tilde{b}^{\alpha \beta}+2 H \rho\left(\rho F_{\rho}+K F_{K}\right) \\
& +\rho\left(2 H^{2}-K\right) F_{H}
\end{aligned}
$$

and

$\left(\rho^{2} F_{\rho}\right)_{, \alpha}+\rho\left(F_{K} K_{, \alpha}+F_{H} H_{, \alpha}\right)=0$, 
respectively, in which $\Delta(\cdot)=(\cdot)_{; \alpha \beta} a^{\alpha \beta}$ is the surface Laplacian, also known as the Beltrami operator,

$\tilde{b}^{\alpha \beta}=2 H a^{\alpha \beta}-b^{\alpha \beta}$

is the cofactor of the curvature, $F\left(\rho, H, K ; \theta^{\mu}\right)$ is the free energy per unit mass,

$H=\frac{1}{2}\left(\kappa_{\nu}+\kappa_{t}\right), \quad K=\kappa_{\nu} \kappa_{t}-\tau^{2}$,

respectively, are the mean and Gaussian curvatures of the surface, and $p$ is the net lateral pressure on the surface in the direction of its orientation $\mathbf{n}$. The subscripts $\rho, H, K$ refer to partial derivatives of the energy with respect to the indicated variables. Equations (4) and (5) together constitute the generalization of the well known shape equation (Ou-Yang et al. 1999) to films with non-uniform properties (Steigmann 1999a).

The list $\{\rho, H, K\}$ of independent variables in the freeenergy function is dictated by material symmetry requirements pertaining to the fluid-like response characteristic of biomembranes. The underlying concept is developed fully in (Steigmann 1999a).

It is well known that it is energetically favorable for membranes to preserve surface area as they deform (Evans and Skalak 1980). To account for this, we impose $J=1$ as a local constraint and replace $F$ by

$F\left(\rho, H, K ; \theta^{\mu}\right)=\bar{F}\left(H, K ; \theta^{\mu}\right)-\bar{\gamma}\left(\theta^{\mu}\right) J$,

where $\bar{F}$ is a constitutive function for the film and $\bar{\gamma}$ is a constitutively-indeterminate Lagrange-multiplier field. The latter takes whatever values that may be required by the equations of equilibrium and any subsidiary conditions. To justify (8) we appeal to a rigorous treatment of constraints in shells (Steigmann 1999b) which subsumes the present framework. Further, we have used the constraint to equate $\rho$ with $\rho_{0}\left(\theta^{\mu}\right)$ and absorbed the latter function into the explicit coordinate dependence appearing in $\bar{F}$. The equivalent expression

$F\left(\rho, H, K ; \theta^{\mu}\right)=\bar{F}\left(H, K ; \theta^{\mu}\right)-\gamma\left(\theta^{\mu}\right) / \rho$,

where $\gamma=\rho_{0} \bar{\gamma}$, is preferred here as it yields the formula $\gamma=$ $\rho^{2} F_{\rho}$ and hence the interpretation of the Lagrange-multiplier field $\gamma$ as the surface pressure; $-\gamma$ then corresponds to the surface tension.

The free energy per unit area of the film is

$W\left(H, K ; \theta^{\mu}\right)=\rho_{0}\left(\theta^{\mu}\right) \bar{F}\left(H, K ; \theta^{\mu}\right)$,

and we define

$-\lambda=\gamma+W$.

In terms of these, Eqs. (4) and (5) reduce respectively to

$$
\begin{aligned}
p=\Delta & \left(\frac{1}{2} W_{H}\right)+\left(W_{K}\right)_{; \alpha \beta} \tilde{b}^{\alpha \beta}+W_{H}\left(2 H^{2}-K\right) \\
& +2 H\left(K W_{K}-W\right)-2 \lambda H
\end{aligned}
$$

and

$\lambda_{, \alpha}=-\partial W / \partial \theta^{\alpha}$,

where $\partial W / \partial \theta^{\alpha}$ is the derivative with respect to $\theta^{\alpha}$ at fixed values of $H$ and $K$. This vanishes in uniform films, and arises from the explicit dependence of $W$ on the coordinates in the case of a non-uniform film. In the former case Eq. (12), with $\lambda=$ const., is the conventional form of the shape equation. It is interesting that the adjustment required for the extension of the theory to non-uniform films is limited simply to the addition of (13). The variational derivation of these equations is discussed in Sect. 3.

In the present work we consider a simple extension of the Helfrich (1973) model to the case of non-uniform spontaneous curvature. Thus,

$W=k(H-C)^{2}$,

where $k(>0)$ is the (constant) bending modulus and $C\left(\theta^{\alpha}\right)$ is the (variable) spontaneous curvature. Here we assume the function $C\left(\theta^{\alpha}\right)$ to be assigned. In a more general treatment, which we do not pursue here, $C\left(\theta^{\alpha}\right)$ would evolve in accordance with the coupled interplay between membrane shape and the distribution and concentration of proteins.

Proceeding with the expression (14) for the energy density, we find that (13) reduces to

$\lambda_{, \alpha}=2 k(H-C) \partial C / \partial \theta^{\alpha}$,

while the shape equation (12) becomes

$$
\begin{aligned}
& k \Delta(H-C)+2 k(H-C)\left(2 H^{2}-K\right)-2 k H(H-C)^{2} \\
& \quad=p+2 \lambda H .
\end{aligned}
$$

In the remainder of this work we impose $p=0$. This is motivated by empirical observations, pertaining to the systems studied here, indicating that the membrane is sensibly flat (so that $H$ and $K$ vanish) over substantial portions of its area where the protein coat density is negligible (and hence where $C$ vanishes). Equation (16) implies that the pressure vanishes in these portions; and thus, if uniform, everywhere on the film. More generally, a solution to (15) and (16) is then furnished by $H\left(\theta^{\mu}\right)=C\left(\theta^{\mu}\right)$ and $\lambda\left(\theta^{\mu}\right)=0$. If a corresponding surface exists, it achieves the global infimum, namely zero, of the energy

$E=k \int_{\omega}\left[H-C\left(\theta^{\mu}\right)\right]^{2} \mathrm{~d} a$,

and is thus optimal in the sense that any compactly supported displacement is necessarily accompanied by a non-negative change of energy. Existence is contingent on the consistency of the condition $H\left(\theta^{\mu}\right)=C\left(\theta^{\mu}\right)$ with the Gauss and Mainardi-Codazzi equations of differential geometry (Kreyszig 1959) and with operative boundary data. 


\section{Variational approach}

The equilibrium equations (12) and (13) follow directly from the balance laws and constitutive equations for a fluid film with bending resistance (Jenkins 1977a; Steigmann 1999a,b). In contrast, in the procedure adopted universally in the literature, the shape equation is derived as the Euler equation associated with the variational problem of minimizing the energy of a uniform film under appropriate side conditions (Ou-Yang et al. 1999). A recent derivation of this kind, extending the argument from a commonly imposed global area constraint to the local version assumed here, has been presented in Steigmann et al. (2003). This too is restricted to films with properties that are uniform in the sense that the energy density $W$ on $\omega$ does not depend explicitly on the coordinates. In particular, (12) emerges as the Euler equation associated with normal variations (variations in position parallel to $\mathbf{n}$ ) of the energy functional

$E=\int_{\omega} W(H, K) \mathrm{d} a$

In Steigmann et al. (2003) the pressure $p$ appearing in (12) is a (constant) Lagrange multiplier enforcing a global constraint on the volume enclosed by the film, considered there to be a closed surface. However, typically the concern is with circumstances in which the volume-to-area ratio adjusts in response to changes in temperature or osmotic pressure. Because area is conserved, it then follows that volume is not, implying that $p$ is a property of the system and thus not a Lagrange multiplier. In such circumstances (12) and (13) remain valid, but, in general, an associated energy functional may not exist. In any event we impose $p=0$ for the reason discussed in the previous section.

Thus, to extend the procedure adopted in the literature to the present problem, it is of interest to outline the variational derivation of Eqs. (12) and (13) for the case of non-uniform film properties. To this end we compute the variation $\dot{E}$ of the energy induced by a variation $\mathbf{u}\left(\theta^{\alpha}\right)=\dot{\mathbf{r}}$ of the equilibrium position field $\mathbf{r}\left(\theta^{\alpha}\right)$. Special roles are played here by the tangential and normal variations, $u^{\alpha}$ and $u$ respectively, in the general expression

$\mathbf{u}=u^{\alpha} \mathbf{a}_{\alpha}+u \mathbf{n}$.

To allow for the present constraint on area, we consider the augmented energy functional

$E=\int_{\Omega}\left[J W\left(H, K ; \theta^{\alpha}\right)-\mu\left(\theta^{\alpha}\right)(J-1)\right] \mathrm{d} A$, where $\mu$ is a Lagrange multiplier field. The variation induced by $\mathbf{u}$ is

$\dot{E}=\int_{\omega}[\dot{W}+(W-\mu) \dot{J} / J] \mathrm{d} a$,

where

$\dot{W}=W_{H} \dot{H}+W_{K} \dot{K}$.

We note that, while the augmented functional may be considered to depend on both the position and Lagrange multiplier, regarded as independent fields, variation with respect to the latter merely returns the constraint and so need not be made explicit.

For tangential variations, formulae developed in Steigmann et al. (2003) yield

$\dot{J} / J=u_{; \alpha}^{\alpha}, \quad \dot{H}=u^{\alpha} H_{, \alpha} \quad$ and $\quad \dot{K}=u^{\alpha} K_{, \alpha}$,

wherein $J, H$ and $K$ are equilibrium fields. These furnish

$(W-\mu) \dot{J} / J=\left[(W-\mu) u^{\alpha}\right]_{; \alpha}-u^{\alpha}(W-\mu)_{, \alpha}$,

which may be combined with (22), (23) 2,3 and Stokes' theorem to obtain

$$
\begin{aligned}
\dot{E}= & \int_{\omega} u^{\alpha}\left(W_{H} H_{, \alpha}+W_{K} K_{, \alpha}-W_{, \alpha}+\mu_{, \alpha}\right) \mathrm{d} a \\
& +\int_{\partial \omega}(W-\mu) u^{\alpha} v_{\alpha} \mathrm{d} s,
\end{aligned}
$$

where $v_{\alpha}$ are the covariant components of the exterior unit normal to the edge $\partial \omega$, lying in the tangent plane of $\omega$, as it is traversed in the direction of increasing arclength $s$. The associated Euler equation is equivalent to the vanishing of the parenthetical term in the first integral. To explore its implications, we compute

$W_{, \alpha}=W_{H} H_{, \alpha}+W_{K} K_{, \alpha}+\partial W / \partial \theta^{\alpha}$,

where, as in (13), the partial derivative on the right is due to the explicit coordinate dependence in the function $W$. This arises from the non-uniformity of the film properties in the present context. Accordingly, the relevant Euler equation is expressible in the form $\mu_{, \alpha}=\partial W / \partial \theta^{\alpha}$, and is seen, on identification of $\mu$ with $-\lambda$, to be identical to (13).

Under normal variations we have (Steigmann et al. 2003)

$\dot{J} / J=-2 H u, \quad 2 \dot{H}=\Delta u+u\left(4 H^{2}-2 K\right)$ and

$\dot{K}=2 K H u+\left(\tilde{b}^{\alpha \beta} u_{, \alpha}\right)_{; \beta}$. 
With some effort these lead to (Steigmann et al. 2003)

$$
\begin{aligned}
\dot{E}=\int_{\omega} & u\left[\Delta\left(\frac{1}{2} W_{H}\right)+\left(W_{K}\right)_{; \beta \alpha} \tilde{b}^{\beta \alpha}+W_{H}\left(2 H^{2}-K\right)\right. \\
& \left.+2 K H W_{K}-2 H(W-\mu)\right] \mathrm{d} a \\
& +\int_{\partial \omega}\left[\frac{1}{2} W_{H} \nu^{\alpha} u_{, \alpha}-\frac{1}{2}\left(W_{H}\right)_{, \alpha} \nu^{\alpha} u+W_{K} \tilde{b}^{\alpha \beta} v_{\beta} u_{, \alpha}\right. \\
& \left.-\left(W_{K}\right)_{, \alpha} \tilde{b}^{\alpha \beta} v_{\beta} u\right] \mathrm{d} s .
\end{aligned}
$$

The relevant Euler equation is given by the vanishing of the bracketed term in the first integral. This is seen, in the case $p=0$ and again upon identification of $\mu$ with $-\lambda$, to be identical to the shape equation (12). The integrals over $\partial \omega$ in (25) and (28) are absent in closed films without boundary. We defer discussion of these terms to Sects. 5 and 6.

It has been shown by Agrawal and Steigmann (2008) that a necessary condition for an equilibrium state to be energy minimizing is

$\frac{1}{4} W_{H H}+\varsigma W_{H K}+\varsigma^{2} W_{K K}>0$

at all points of the film, where

$\varsigma=\tilde{b}^{\alpha \beta} b_{\alpha} b_{\beta}$,

and the $b_{\beta}$ are arbitrary subject to the normalization condition $a^{\alpha \beta} b_{\alpha} b_{\beta}=1$. Thus, $\varsigma$ is bounded between the largest and smallest eigenvalues of the cofactor of the curvature on the equilibrium surface. These bounds coincide, by virtue of (6), with the principal curvatures on the film. In principle, inequality (29) is a restriction on energy minimizers. However, for the quadratic energy density considered here, it effectively reduces to a constitutive inequality. To see this, we substitute (14) and find that (29) reduces to the requirement $k>0$, which justifies the assumption made in connection with (14). This inequality does not entail any restriction on the state of the film. Accordingly, if it holds in an energy-minimizing state, then it holds in all states, and, in that sense, imposes a restriction of a constitutive nature; that is, a restriction on the function $W$.

\section{Surfaces of revolution}

We consider axisymmetric surfaces parameterized by meridional arclength $s$ and azimuthal angle $\theta$. Let $r(s)$ be the radius from the axis of symmetry and $z(s)$ be the elevation above a base plane (see Fig. 1). Meridians and parallels of latitude are the curves on which $\theta$ and $s$, respectively, are constant. We select surface coordinates $\theta^{1}=s$ and $\theta^{2}=\theta$, and identify the vector $\boldsymbol{v}$ of (2) with the unit tangent to a meridian. Further, because $s$ measures arclength along meridians, we have

$\left(r^{\prime}\right)^{2}+\left(z^{\prime}\right)^{2}=1$,

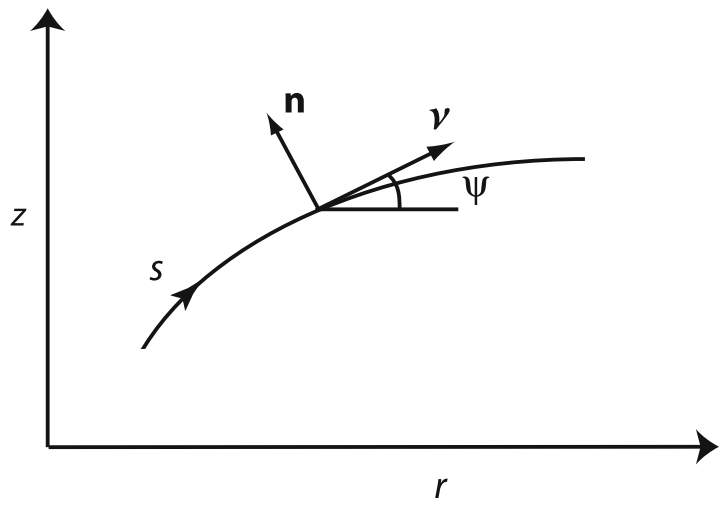

Fig. 1 Meridian of a surface of revolution

where, here and henceforth, ()$^{\prime}=d() / d s$. This implies that

$r^{\prime}(s)=\cos \psi$ and $z^{\prime}(s)=\sin \psi$,

where $\psi(s)$ is the angle made by $\boldsymbol{v}$ with the radial direction.

The metric and dual metric are $\left(a_{\alpha \beta}\right)=\operatorname{diag}\left(1, r^{2}\right)$ and $\left(a^{\alpha \beta}\right)=\operatorname{diag}\left(1, r^{-2}\right)$, respectively, and the covariant components of curvature are $\left(b_{\alpha \beta}\right)=\operatorname{diag}\left(\psi^{\prime}, r \sin \psi\right)$ (Agrawal and Steigmann 2008). Combining these with (1), (2) and (32), we derive

$\kappa_{v}=\psi^{\prime}, \quad \kappa_{t}=r^{-1} \sin \psi$ and $\tau=0$.

The sum of the normal curvatures is twice the mean curvature $H(s)$. This furnishes the differential equation

$r \psi^{\prime}=2 r H-\sin \psi$.

Their product is the Gaussian curvature $K(s)$; thus,

$K=H^{2}-\left(H-r^{-1} \sin \psi\right)^{2}$.

For the free-energy function (14), the shape equation (16) simplifies, in the absence of lateral pressure, to (see also Agrawal and Steigmann 2008)

$\begin{aligned} L^{\prime}= & 2 r\left\{H\left[k(H-C)^{2}+\lambda\right]-k(H-C)\right. \\ & \left.\times\left[H^{2}+\left(H-r^{-1} \sin \psi\right)^{2}\right]\right\},\end{aligned}$

where

$L=\frac{1}{2} r\left(W_{H}\right)^{\prime}=k r(H-C)^{\prime}$.

We assume the spontaneous curvature to be an assigned function $C(s)$ of the meridional arclength. The remaining equilibrium equation (13), yields $\partial \lambda / \partial \theta=0$ and

$\lambda^{\prime}=2 k(H-C) C^{\prime}$.

The system to be solved thus consists of the six Eqs. (32) $1,2,(34),(36)$, (37) and (38), for the six unknowns $r, z, \psi$, $H, \lambda$ and $L$. Four of the boundary conditions are of the geometric type. Thus,

$r(0)=r_{0}, \quad \psi(0)=\psi_{0}, \quad z(0)=z_{0} \quad$ and $\quad \psi(S)=\psi_{S}$, 
where the right-hand sides and the value of $S$ are to be specified. We append additional boundary conditions based on equilibrium considerations. These derive from the fact, explained in detail in (Agrawal and Steigmann 2008), that the limits of the transverse shear force, per unit length of arc, must vanish as the equator is approached whenever the latter is a plane of reflection symmetry (see also Jenkins 1977b). It must also vanish at a pole; that is, at a point of the membrane lying on the axis of axisymmetry. In both cases this is equivalent to the requirement

$L=0$

at the appropriate value(s) of arclength. Because the pole does not belong to the boundary when the film is regarded as a two-dimensional surface, the latter condition does not arise as a natural boundary condition in the formulation discussed in Sect. 3. However, the pole does constitute a boundary in a one-dimensional formulation based on the use of arclength as independent variable. If the variational problem is rephrased in this setting, then (40) emerges as a natural boundary condition associated with the unrestrained variation of the $z$-coordinate of the pole (Jenkins 1977b). Alternatively, it may be derived from a direct analysis of the equilibrium of a sector of the surface containing the pole, in the limit as the size of the sector shrinks to zero (Agrawal and Steigmann 2008).

To non-dimensionalize the equations we introduce a positive constant $H_{0}$ having the dimensions of curvature. The dimensionless arclength, radius and height are

$t=s H_{0}, \quad x=r H_{0} \quad$ and $\quad y=z H_{0}$,

respectively, and the dimensionless mean and spontaneous curvatures are

$\bar{H}=H / H_{0}$ and $\quad \bar{C}=C / H_{0}$.

Equations (32) and (34) may be written

$\dot{x}=\cos \psi, \quad \dot{y}=\sin \psi \quad$ and $\quad x \dot{\psi}=2 x \bar{H}-\sin \psi$,

where ()$^{\circ}=H_{0}^{-1}()^{\prime}$ is the derivative with respect to $t$. Finally, Eqs. (36) and (38) may be reduced, respectively, to

$$
(\bar{L})^{\cdot}=2 x\left\{\bar{H}(\bar{W}+\bar{\lambda})-(\bar{H}-\bar{C})\left[\bar{H}^{2}+\left(\bar{H}-x^{-1} \sin \psi\right)^{2}\right]\right\},
$$

and

$(\bar{\lambda})^{\cdot}=2(\bar{H}-\bar{C})(\bar{C})^{\prime}$,

where

$\bar{L}=\left(k H_{0}\right)^{-1} L=x(\bar{H}-\bar{C})^{,}, \quad \bar{\lambda}=\left(k H_{0}^{2}\right)^{-1} \lambda$ and

$\bar{W}=\left(k H_{0}^{2}\right)^{-1} W=(\bar{H}-\bar{C})^{2}$

is the dimensionless energy.

\section{Nuclear pores}

Nuclear pores mediate traffic into and out of the cell nucleus and generate the two-leaf structure of the nuclear envelope. They are accompanied by a local distribution of proteins concentrated near the pore opening which appear to facilitate pore formation (Drummond and Wilson 2002). To describe an isolated nuclear pore, we assign a hypothetical spontaneous curvature distribution having the Gaussian form (see Fig. 2)

$\bar{C}(t)=\beta \exp \left(-\alpha t^{2}\right)$,

where $\alpha$ and $\beta$ are positive constants. Thus, the concentration of proteins, and hence the intensity of the spontaneous curvature, is assumed to be greatest at the equatorial plane where $t=0$.

The interior equilibrium equations (44) and (45) are satisfied identically if $\bar{H}(t)=\bar{C}(t)$ and $\bar{\lambda}(t)=0$. Clearly the zero-shear-force condition (40) at the equator is then automatically satisfied. Equations (43) 1,2,3 constitute the remaining system, which is integrated numerically using Matlab (ODE solver 'bvp4c') with $\psi_{0}=\pi / 2, z_{0}=0$ and $\psi(T)=0$, where $T=S H_{0}$. A sequence of values of $T$ is chosen, corresponding to a succession of domains of increasing total arclength. The sequence is terminated when the difference between succeeding solutions is negligible on the domain of the last-solved boundary-value problem. This effectively models an unbounded domain, or, what is equivalent, an isolated pore sufficiently removed from its neighbors such that the effects of interaction may be ignored. Further, if $\partial \omega$ is identified with the circle $t=T$, then the solution obtained is such that the boundary terms in the expressions (25) and (28) for the variation of the energy vanish identically for the consi-

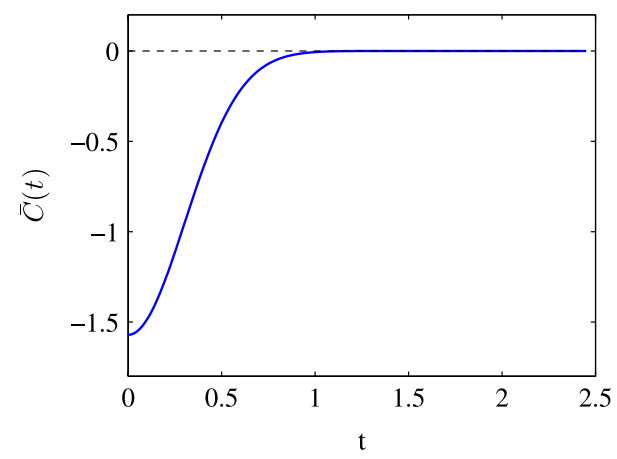

Fig. 2 Spontaneous curvature distribution in the nuclear pore $(\alpha=5.5$ and $\beta=-\pi / 2$ ) 


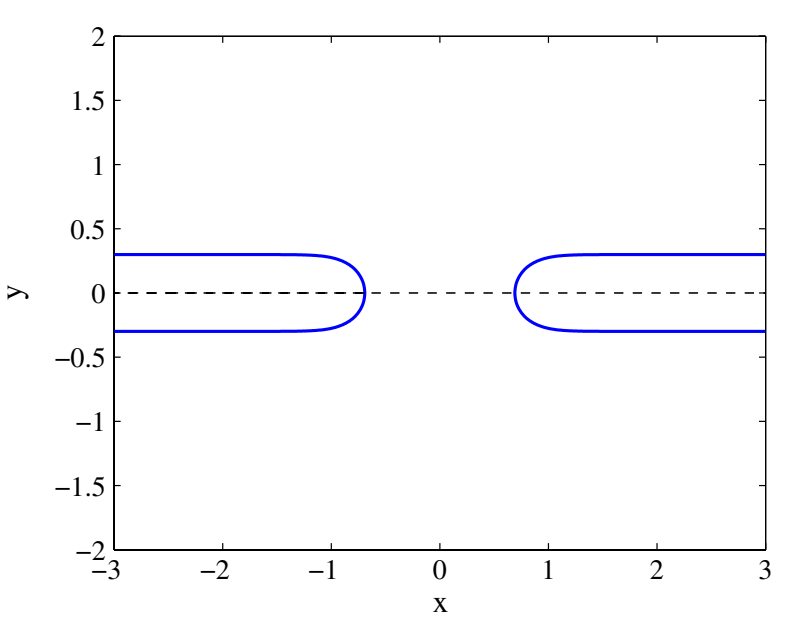

Fig. 3 Predicted shape of the nuclear pore

dered free-energy function. Accordingly, it is an equilibrium state in the sense that it renders the total energy functional stationary.

The solution is depicted in Fig. 3, which shows the shape of the deformed meridian obtained by adjusting the parameters in (48) $(\alpha=5.5, \beta=-\pi / 2)$ to fit physiological data on the ratio of membrane height to pore diameter. The value of the constant $H_{0}$ may be adjusted to fit some feature of the meridian, such as the equatorial pore radius $r(0)=x(0) / H_{0}$, to actual dimensions. We find that $\beta H_{0}=-0.024 \mathrm{~nm}^{-1}$ yields a pore radius of approximately $45 \mathrm{~nm}$ and height (distance from the equatorial plane) of approximately $20 \mathrm{~nm}$, in agreement with observations on actual pores (Beck et al. 2004; Margalit et al. 2005). The solution obtained has the mean curvature equal everywhere to the spontaneous curvature and is therefore energetically optimal.

\section{Vesicle formation during endocytosis}

The present model may also be used to simulate endocytosis; i.e., the process of protein-assisted vesicle formation in

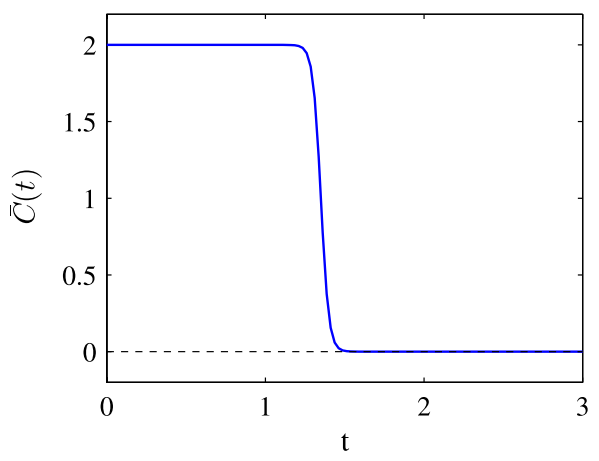

Fig. 4 Spontaneous curvature distribution in endocytosis $(\alpha=20.0$, $\beta=2.0$ and $t_{0}=1.3$ )

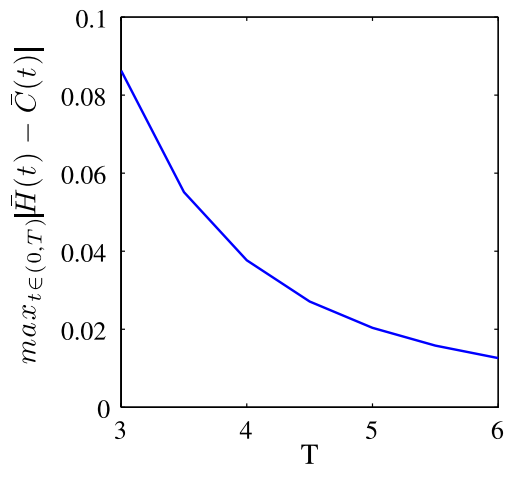

Fig. 5 Convergence of $\bar{H}(t)$ to $\bar{C}(t)$ with increasing $T$

lipid membranes. Here we model the stage from initiation to bud formation prior to fission. In this process, a protein coat such as clathrin (Alberts et al. 2002; Boal 2002) forms on part of the membrane, inducing an essentially uniform distribution of natural curvature over the coated region. This is again modeled as a distribution of spontaneous curvature. An appropriate function for this problem is (see Fig. 4)

$\bar{C}(t)=\frac{\beta}{2}\left\{1-\tanh \left[\alpha\left(t-t_{0}\right)\right]\right\}$.

This models the abrupt transition of protein concentration (Boal 2002) from a nearly constant value in the coated region to zero in the uncoated region. The progression of endocytosis is simulated by adjusting the parameter $t_{0}$, with larger values corresponding to more of the membrane being coated and hence naturally curved.

The solution procedure is as described in the previous section, except that the full set of Eqs. (43)-(47) is integrated subject to the data

$x(0)=0, \quad \psi(0)=0, \quad y(0)=0 \quad$ and $\quad \bar{L}(0)=0$

at the pole $(t=0)$, together with

$\psi(T)=0$ and $\bar{\lambda}(T)=0$.

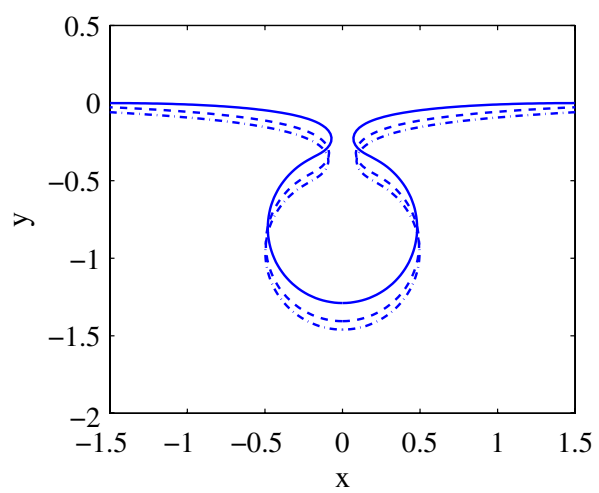

Fig. 6 Vesicle shapes for $T=3.0$ (solid line), $T=4.5$ (dashed line), and $T=6.0$ (dashed-dot line) 
Fig. 7 a Shallow

clathrin-coated vesicle observed by Perry and Gilbert (1979, reproduced by permission of the Company of Biologists), and b present simulation obtained with $t_{0}=0.3$ (a)

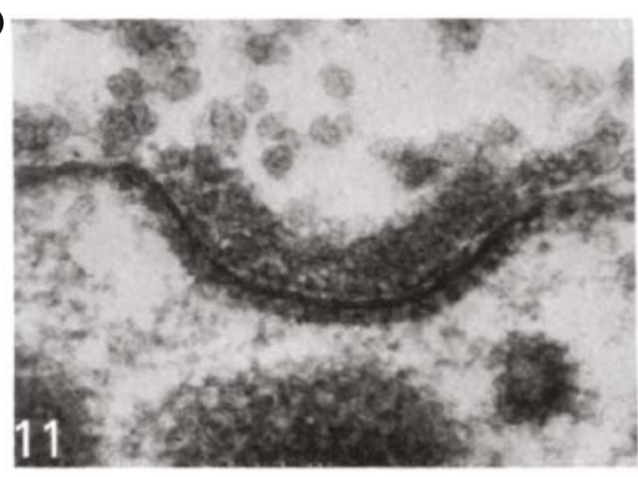

(a)

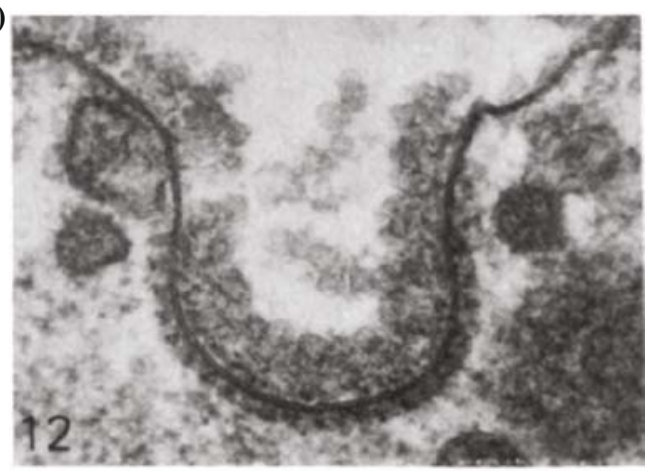

(a)

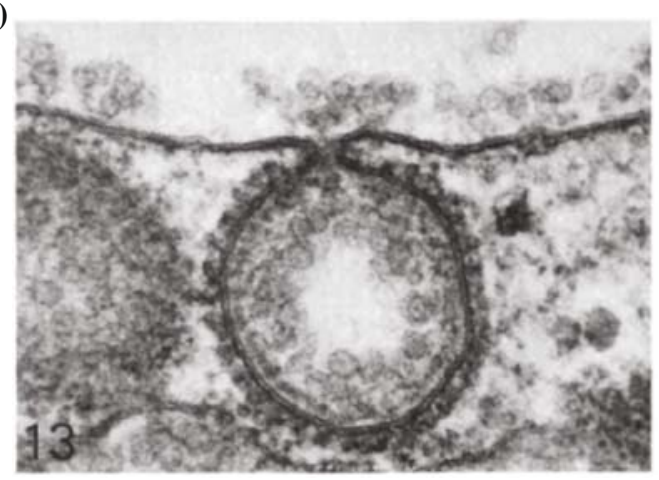

(b)

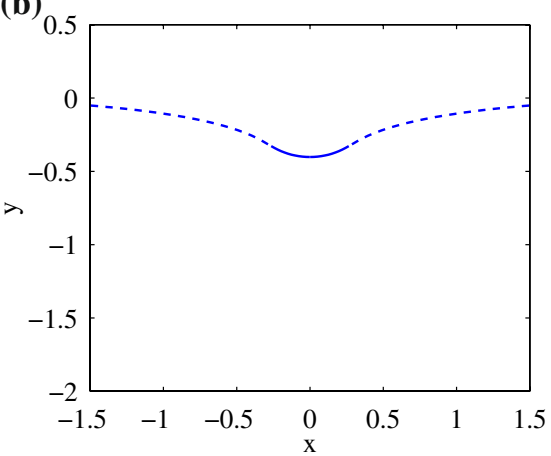

(b)

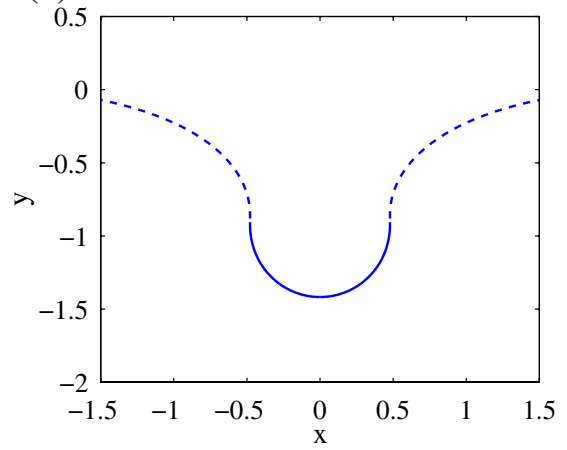

(b)

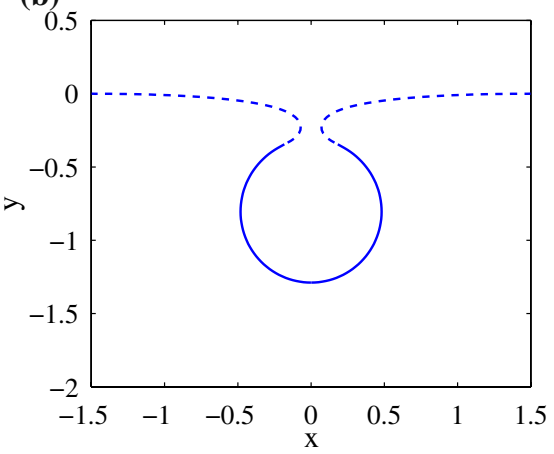

Fig. 9 a Final stage of clathrin-coated vesicle prior to fission, observed by Perry and Gilbert (1979, reproduced by permission of the Company of Biologists), and $\mathbf{b}$ present simulation obtained with $t_{0}=1.3$
Fig. 8 a Intermediate shape of Company of Biologists), and b present simulation obtained with $t_{0}=0.8$ 
that $\max _{t \in(0, T)}|\bar{H}(t)-\bar{C}(t)|$ decays to zero as $T$ increases (see Fig. 5). This in turn implies that the edge asymptotically approaches a state of ease in the sense described above. We also observe that the shape of the surface, as described by $\{x(t), y(t)\}$, effectively converges as $T$ increases. This conclusion follows from Fig. 6, which depicts a sequence of meridians for the parameter values $\alpha=20.0, \beta=2.0$ and $t_{0}=1.3$.

Figures 7, 8 and 9 show photo-micrographs of endocytosis (Perry and Gilbert 1979) alongside membrane shapes computed from the present model using the same values of $\alpha$ and $\beta$, but with $t_{0}=0.3,0.8,1.3$ respectively, corresponding to protein coats of successively increasing size. The coated regions are the solid portions of the meridional curves. The shapes shown correspond to $T=3.0$. It is found that $\beta H_{0}=0.0036 \mathrm{~nm}^{-1}$ yields a vesicle diameter of approximately $280 \mathrm{~nm}$, which is the average observed in clathrin-coated vesicles (Perry and Gilbert 1979).

We emphasize that the finite-domain $(T<\infty)$ problem furnishes a meaningful model of endocytosis in its own right. However, the total energy associated with a particular fixed domain asymptotically approaches the infimum of the energy, associated with that domain, at large $T$. For finite $T$ our solutions are equilibrium states, but we do not know if they are energetically optimal.

\section{Conclusions}

A quantitative model of protein-mediated morphology has been developed on the basis of the conventional continuum theory of lipid membranes, extended to account for non-uniform properties. This extension is needed to accommodate the effects of non-uniformly distributed proteins that interact with the bilayer in such a way as to induce a variable natural, or spontaneous, curvature field. The model accounts for protein type and distribution indirectly through the spontaneous curvature. Solutions obtained in the specialization to axisymmetry are found to be robust in the sense of being energetically optimal relative to all competing configurations, not merely those that preserve axisymmetry. Further, these yield quantitative information for morphologies associated with nuclear pores and endocytosis.

A drawback of the present work is that the distribution of spontaneous curvature must be specified. In some circumstances it may be more natural to regard this distribution as evolving with membrane shape rather than as specified data. We envisage a model for the diffusion of densely distributed proteins on curved surfaces. In that setting, the concentration of proteins would generate a distribution of spontaneous curvature which would affect the local free-energy function and consequent membrane shape via the equations of equilibrium given here. In turn, shape would be expected to influence the distribution of local protein concentration as diffusion proceeds.

Acknowledgments We thank an anonymous referee for a number of valuable comments leading to substantial improvements in the manuscript.

\section{References}

Agrawal A, Steigmann DJ (2008) Coexistent fluid-phase equilibria in biomembranes with bending elasticity. J Elast 93:63-80

Alberts B, Johnson A, Lewis J, Raff M, Roberts K, Walter P (2002) Molecular biology of the cell, 4th edn. Garland Science, New York

Beck M, Förster F, Ecke M, Plitzko JM, Melchior F, Gerisch G, Baumeister W, Medalia O (2004) Nuclear pore complex structure and dynamics revealed by cryoelectron tomography. Science 306: $1387-1390$

Boal D (2002) Mechanics of the Cell. Cambridge University Press, Cambridge

Drummond SP, Wilson KL (2002) Interference with the cytoplasmic tail of 'gp210' disrupts close apposition of nuclear membranes and blocks nuclear pore dilation. J Cell Biol 158:53-62

Evans EA, Skalak R (1980) Mechanics and thermodynamics of biomembranes. CRC Press, Boca Raton

Helfrich W (1973) Elastic properties of lipid bilayers: theory and possible experiments. Z Naturforsch 28:693-703

Jenkins JT (1977a) Static equilibrium configurations of a model red blood cell. J Math Biol 4:149-169

Jenkins JT (1977b) The equations of mechanical equilibrium of a model membrane. SIAM J Appl Math 32:755-764

Kim KS, Neu J, Oster G (1998) Curvature-mediated interactions between membrane proteins. Biophys J 75:2274-2291

Kreyszig E (1959) Differential geometry. University of Toronto Press, Toronto

Margalit A, Vlcek S, Gruenbaum Y, Foisner R (2005) Breaking and making of the nuclear envelope. J Cell Biochem 95:454-465

Ou-Yang Z-C, Liu J-X, Xie Y-Z (1999) Geometric methods in the elastic theory of membranes in liquid crystal phases. World Scientific, Singapore

Perry MM, Gilbert AB (1979) Yolk transport in the ovarian follicle of the hen (Gallus domesticus): Lipoprotein-like particles at the periphery of the oocyte in the rapid growth phase. J Cell Sci 39: 257-272

Steigmann DJ (1999a) Fluid films with curvature elasticity. Arch Rational Mech Anal 150:127-152

Steigmann DJ (1999b) On the relationship between the Cosserat and Kirchhoff-Love theories of elastic shells. Math Mech Solids 4:275-288

Steigmann DJ, Baesu E, Rudd RE, Belak J, McElfresh M (2003) On the variational theory of cell-membrane equilibria. Interfaces Free Boundaries 5:357-366 